\title{
ON THE OSCILLATION OF DIFFERENTIAL TRANSFORMS OF EIGENFUNCTION EXPANSIONS
}

\author{
BY
}

\author{
C. L. PRATHER AND J. K. SHAW
}

\begin{abstract}
This paper continues the study of Pólya and Wiener, Hille and Szegö into the connections between the oscillation of derivatives of a real function and its analytic character. In the present paper, a Sturm-Liouville operator $L$ is applied successively to an infinitely differentiable function which admits a certain eigenfunction expansion. The eigenfunction expansion is assumed to be "conservative", in the sense of Hille. Several theorems are given which link the frequency of oscillation of $\left(L^{k} f\right)(x)$ to the size of the coefficients of $f(x)$, and thus to its analytic character.
\end{abstract}

1. Introduction. Let $L$ be the second order linear differential operator $L y=$ $p_{2}(x) y^{\prime \prime}+p_{1}(x) y^{\prime}+p_{0}(x) y$, and consider the solutions on an interval $(a, b)$ of the problem

$$
L y=-\lambda y .
$$

The parameter $\lambda$ is complex-valued, and $(a, b)$ is an interval of regular points of $L$, although the endpoints may be singular. We list our specific assumptions on the operator and its coefficients in $\$ 2$, but we mention here that (1.1) will include most regular or singular Sturm-Liouville operators, and those equations giving rise to the classical orthogonal polynomials.

It is often convenient to write (1.1) in its selfadjoint form. This comes about by multiplying (1.1) by the integrating factor

$$
P(x)=\frac{1}{p_{2}(x)} \exp \left\{\int_{\gamma}^{x} \frac{p_{1}(t)}{p_{2}(t)} d t\right\} \quad(\gamma, x \in(a, b))
$$

and then replacing (1.1) by

$$
\tilde{L} y=\left(P(x) p_{2}(x) y^{\prime}\right)^{\prime}+P(x) p_{0}(x) y=-P(x) \lambda y .
$$

Let us suppose that (1.1) has been equipped with boundary conditions of some sort, so that a complete orthonormal set of eigenfunctions $\left\{u_{n}(x) ; \mu_{n}\right\}_{n=1}^{\infty}$ results. That is, for each $n, u_{n}(x)$ satisfies

$$
\tilde{L} u_{n}(x)=-\mu_{n} P(x) u_{n}(x) \quad\left(L u_{n}(x)=-\mu_{n} u_{n}(x)\right)
$$

together with its boundary conditions (types of boundary conditions encountered in this paper, along with assumptions on the eigenvalues $\mu_{n}$, are also detailed in $\$ 2$

Received by the editors August 23, 1982.

1980 Mathematics Subject Classification. Primary 42C15, 34B25; Secondary 30B50.

Key words and phrases. Eigenfunction expansion, iterates of operators, sign changes.

(C)1983 American Mathematical Society $0002-9947 / 83 \$ 1.00+\$ .25$ per page 
below). Suppose we have the eigenfunction expansion of a function $f(x)$,

$$
f(x)=\sum_{n=1}^{\infty} f_{n} u_{n}(x), \quad a<x<b,
$$

whose generalized Fourier coefficients are so restricted that the successive iterates $L f, L^{2} f, \ldots$ of the differential transform $L$ are computable from termwise application in (1.4), i.e.

$$
\left(L^{k} f\right)(x)=\sum_{n=1}^{\infty}\left(-\mu_{n}\right)^{k} f_{n} u_{n}(x), \quad k=1,2,3, \ldots
$$

Now typically, $u_{m}(x)$ has $m$ zeros in $(a, b)$. At the same time, if the coefficients $f_{n}$ are suitably behaved, we may choose $k$ in such a way that a certain term $\left(-\mu_{m}\right)^{k} f_{m} u_{m}(x)$ is the dominant one in (1.5). In this situation we may expect $\left(L^{k} f\right)(x)$ to experience oscillation, in fact to have $m$ zeros, or sign changes, in $(a, b)$. On the other hand, if (1.4) is a finite series, ending with term $f_{M} u_{M}(x)$ say, and $0<\mu_{1}<\mu_{2}<\cdots<\mu_{M}$, then $\left(-\mu_{M}\right)^{k} f_{M} u_{M}(x)$ will so dominate $(1.5)$ that $\left(L^{k} f\right)(x)$ will have $M$ zeros, and no more, for all large $k$. Thus, the coefficients $f_{n}$ cannot be too small if unbounded frequency of oscillation is to occur. This brings us to the first of two general problems we study in this paper.

(P1) How large must the magnitudes $\left|f_{n}\right|$ be and, regarding $k=k_{m}$ as dependent on $m$, how large must $k_{m}$ be taken in order that $\left(L^{k_{m}} f\right)(x)$ have at least $m$ sign changes in $(a, b)$ for infinitely many $m$ ?

This question has its origin in the classical works of G. Pólya and N. Wiener [10], E. Hille [5] and G. Szegö [18]. In their study of the zeros of successive derivatives of periodic functions, Pólya and Wiener used Fourier series methods to prove the following.

Theorem A (Polya AND Wiener [10, p. 252]). If $f(x)=\sum_{n=-\infty}^{\infty} c_{n} e^{i n x}\left(c_{-n}=\bar{c}_{n}\right)$ is an infinitely differentiable periodic function and

$$
\limsup _{n \rightarrow \infty}\left|c_{n}\right| e^{n \gamma}=\infty
$$

for some $\gamma>0$, then there is a positive integer $g$ so that $f^{(k)}(x)$ has, for an infinity of values of $k$, more than $(k / g)^{1 / 2}$ changes of sign in a period.

In the context of (Pl) we would say that $k_{m}=g^{2}$. It happens that the integers $k$ constructed in the proof of Theorem $A$ are even, and so the result may be interpreted as a statement about the successive iterates of the differential operator $L y=y^{\prime \prime}$. Taking this point of view, Hille [5] considered the sign changes of the successive transforms (1.5), with $L$ a general second order operator. His principal result [5, p. 482] is the converse to the obvious fact mentioned above for finite expansions. He proves that if (1.4) is a "conservative system" (see §2) and the inferior limit as $k \rightarrow \infty$ of the number of sign changes of $\left(L^{k} f\right)(x)$ is finite, then (1.4) is necessarily a finite series. This extends to the setting of differential operators Theorem I of Pólya and Wiener [10, p. 249]: if $f(x)$ is infinitely differentiable and periodic, and if the number of sign changes of $f^{(k)}(x)$ in a period is bounded as $k \rightarrow \infty$, then $f(x)$ is a trigonometric polynomial. 
Theorem $\mathbf{A}$ is actually the more difficult half of a better-known result (Theorem III of [10]) linking the oscillation of derivatives of a periodic function with its analytic character. Pólya and Wiener note that the condition

$$
\limsup _{n \rightarrow \infty}\left|c_{n}\right| e^{n \gamma}<\infty
$$

for all $\gamma>0$, implies that the Fourier series for $f(x)$ converges in the entire complex plane. Putting this together with the contrapositive of Theorem A, we obtain their Theorem III [10, p. 249]: if the number of sign changes of $f^{(k)}(x)$ in a period is $o\left(k^{1 / 2}\right), k \rightarrow \infty$, then $f(x)$ is necessarily an entire function. A new proof of this, plus an analogous result for iterates of Legendre's operator, and even further extensions, were given by $\mathrm{G}$. Szegö $[\mathbf{1 8}, 19]$.

Noting that (1.6) and (1.7) are direct alternatives, and returning to Hille's differential operator setting, we arrive at the second general problem studied here. Suppose the eigenfunctions $u_{n}(x)$ can be continued analytically off of $(a, b)$ into a region $\Omega$ in the complex plane containing $(a, b)$.

(P2) How small must the magnitudes $\left|f_{n}\right|$ be in order that (1.4) converge to an analytic function in $\Omega$ ?

In resolving (P1) and (P2) we accomplish the main purpose of this paper, which is to extend Theorem III of Pólya and Wiener to the framework of operators and conservative systems of Hille. It turns out that the growth rates of $\left|f_{n}\right|$ in (P1) and (P2) are again complementary, and revolve around the condition (cf. [5, p. 496])

$$
\lim \sup \left|f_{n}\right| e^{\gamma \mu_{n}^{\prime}=}=\infty, \quad \gamma>0 .
$$

The value of $k_{m}$ in $(\mathrm{Pl})$ will have to be larger than either $\mu_{m}$ or $\mu_{m}^{2}$, depending on certain technical matters, but in the main cases of interest the frequency of oscillation of $\left(L^{k} f\right)(x)$, under condition (1.8), will be more than $k^{1 /(2+\varepsilon)}$, for arbitrary $\varepsilon>0$ and infinitely many $k$. Very roughly, our principal result will say that for "conservative systems", with eigenvalues $\mu_{n}$ such that either $\mu_{n} \cong n$ or $\mu_{n} \cong n^{2}$, and with eigenfunctions $u_{n}(x)$ analytic in a region $\Omega$ containing $(a, b)$, functions $f(x)$ whose iterates $\left(L^{k} f\right)(x)$ have fewer than $k^{1 /(2+\varepsilon)}$ sign changes in $(a, b)$ are necessarily analytic in $(a, b)$.

In particular, we have a generalization of the following result for orthogonal polynomials which is stated, without proof, in [5].

TheOREM B (Hille [5, p. 496]). Let (1.4) be the expansion of an "admissible" (see §2) function $f(x)$ in either Legendre, Jacobi, Hermite-Weber or Laguerre functions. Assume that the number of sign changes of $\left(L^{k} f\right)(x)$ is $o\left(k^{1 / 2}\right)$ for all large $k$. Then $f(x)$ is an entire function.

In the following section we summarize the axioms of Hille's conservative systems, and we state our basic theorems. Our treatment of (P1) is broken down into two cases, and these occupy $\$ \S 3-4$. Problem (P2) is considered in $\$ 5$.

Some mention should be made of the set of "converse problems" to the one we take up here. Instead of assuming information about the sign changes of $\left(L^{k} f\right)(x)$ 
and deducing analyticity, one could take an assumed analytic eigenfunction expansion $f(x)$ and study the location of the zeros of $\left(L^{k} f\right)(x)$. In particular, the structure of the so-called "final set" of $f(x)$ with respect to the operator $L$ has been investigated. The final set $S(f, L)$ of $f$ with respect to $L$ is defined by saying $z \in S(f, L)$ if every neighborhood of $z$ contains zeros of infinitely many iterates $\left(L^{k} f\right)(x)$. For derivatives, instead of differential operators, this notion is classical (see Pólya's survey paper [9]). The present authors have recently examined various aspects of final set theory in $[\mathbf{1 6}, \mathbf{1 7}]$. Final sets for some nonderivative operators, acting on trigonometric sums and integrals, were determined by Boas and Prather in $[3,11,12]$.

An extension of the results of Pólya and Wiener to Fourier integrals has recently been given by Prather [13]. Other analogues for almost-periodic functions were obtained by Kacnel'son [8]. A special case of the results of the present paper for Hermite and Laguerre expansions was considered in [14].

2. Hypotheses and statements of theorems. We assume the conditions of [5], and will now summarize its specific axioms. While they seem numerous, it will be seen that "conservative systems" include virtually all of the standard boundary value problems of mathematical physics. In return for placing matters on the rather elaborate postulational basis which follows, we shall have a method of proof with wide applications.

To begin with, we require that the coefficients $p_{0}, p_{1}, p_{2}$ of $L$ be real-analytic in $(a, b)$, which is denoted by

$$
\left(\mathrm{A}_{1}\right) \quad p_{m}(x) \in A(a, b), \quad m=0,1,2 \text {, }
$$

and means that $p_{m}(x)$ is real in $(a, b)$ and is the restriction there of an analytic function. Suppose also that

$$
\left(\mathrm{A}_{2}\right) \quad p_{0}(x) \leqslant 0 \text { and } p_{2}(x)>0, \quad a<x<b \text {. }
$$

Hille classifies the operators $L$ into four types, $\mathrm{T}_{\nu}$ for $\nu=1,2,3,4$, according to the behavior of the coefficients $p_{m}(x)$ at the endpoints of $(a, b)$. Type $\mathrm{T}_{1}$ (SturmLiouville) occurs when the $p_{m}(x)$ are analytic at both endpoints $x=a$ and $x=b$, and $p_{2}(a) \neq 0 \neq p_{2}(b)$. Type $\mathrm{T}_{2}$ (Periodic) occurs when, in addition to the conditions of $\mathrm{T}_{1}$, we also have the lead coefficient of (1.2) satisfying $P(a) p_{2}(a)=$ $P(b) p_{2}(b)$. The boundary value problems associated with these two types of operators are the usual "regular" ones. In order to discuss "singular" problems, Hille introduces the quantities $K(x)=P(x) p_{2}(x), G(x, \lambda)=P(x)\left[\lambda-p_{0}(x)\right]$, $Q\left(x, x_{0} ; \lambda\right)=\int_{x_{0}}^{x} G(v, \lambda) d v$ and $R\left(x, x_{0} ; \lambda\right)=\int_{x_{0}}^{x}\left[Q\left(t, x_{0} ; \lambda\right) / K(t)\right] d t$, where $a$ $<x_{0}<b$. If one of the endpoints is a regular point of $L$, we may place $x_{0}$ there. With this notation (1.2) can be written

$$
\left(K(x) y^{\prime}\right)^{\prime}-G(x, \lambda) y=0,
$$

and one has from $\left(\mathrm{A}_{2}\right)$ that $G(x, \lambda)>0$ when $\lambda>0$. This positivity allows Hille [5, Lemma 2, p. 469] to prove that the condition $\limsup _{x \rightarrow b} R\left(x, x_{0} ; \lambda\right)<\infty$ for some $\lambda>0$ is necessary and sufficient for every solution $y(x)$ of (1.2) to be bounded as $x \rightarrow b$ for every $\lambda>0$. A similar criterion may be set at $x=a$. Thus, it is reasonable 
to use the condition $R\left(x, x_{0} ; \lambda\right) \rightarrow \infty$ to determine singular behavior at the endpoints. We say, then, that the operator $L$ is of type $\mathrm{T}_{3}$ (singular) if $R\left(x, x_{0} ; \lambda\right) \rightarrow \infty$, when $x \rightarrow a$ and $x \rightarrow b$, for some $\lambda>0$. If each $p_{n}(x)$ is analytic at $x=b, p_{2}(b) \neq 0$ and $R(x, b ; \lambda) \rightarrow \infty$ when $x \rightarrow a$ and $\lambda>0$, then we say $L$ is of type $\mathrm{T}_{4}$ (semisingular). The roles of $a$ and $b$ may be reversed in the latter case.

It will be convenient to use the notation $\langle a, b\rangle$ to stand for any one of the four alternatives $(a, b),[a, b],(a, b],[a, b)$. To write $p_{m}(x) \in A[a, b)$ will mean that $p_{m}(x)$ is analytic at $x=a$ in addition to $p_{m}(x) \in A(a, b)$, while $p_{n}(x) \in A\langle a, b\rangle$ will indicate any one of four such possibilites.

Associated with each operator type $\mathrm{T}_{\nu}$ is a class $B_{\nu}^{(k)}\{L ;\langle a, b\rangle\}$ of functions of continuity class $C^{(k)}$ which satisfy appropriate boundary conditions. For $k \geqslant 1$,

$$
B_{1}^{(k)}\{L ;[a, b]\}=\left\{f \in C^{2 k}[a, b] ;\left(L^{n} f\right)(a)=\left(L^{n} f\right)(b)=0,0 \leqslant n<k\right\},
$$

and

$$
\begin{aligned}
B_{2}^{(k)}\{L ;[a, b]\}=\left\{f \in C^{(2 k)}\right. & {[a, b] ;\left(L^{m} f\right)(a)=\left(L^{m} f\right)(b), } \\
& \left.\left(D L^{m} f\right)(a)=\left(D L^{m} f\right)(b), 0 \leqslant m<k\right\},
\end{aligned}
$$

where $D$ denotes the ordinary derivative operator. To define $B_{3}^{(k)}$ and $B_{4}^{(k)}$ Hille introduces the solution $y\left(x ; x_{0}, \lambda\right)$ of (1.1) uniquely defined by the initial conditions $y\left(x_{0}\right)=1, y^{\prime}\left(x_{0}\right)=0$, where $a<x_{0}<b$. Again, if an endpoint is regular we may place $x_{0}$ there. The singular boundary class is $B_{3}^{(k)}\{L ;(a, b)\}=\{f \in$ $C^{(2 k)}(a, b) ;\left[\left(L^{n} f\right)(x) / y\left(x ; x_{0}, \lambda\right)\right] \rightarrow 0$ when $x \rightarrow a$ and $x \rightarrow b, 0 \leqslant n<k$, for some $x_{0} \in(a, b)$ and arbitrarily small $\left.\lambda>0\right\}$. As for the semisingular case, we fix nonnegative constants $c_{1}$ and $c_{2}$, not both 0 , and put $B_{4}^{(k)}\{L ;(a, b]\}=\{f \in$ $C^{(2 k)}(a, b] ;\left[\left(L^{n} f\right)(x) / y(x ; b, \lambda)\right] \rightarrow 0$, as $x \rightarrow a$, where $\lambda>0$ is arbitrarily small, and $\left.c_{1}\left(L^{n} f\right)(b)+c_{2}\left(D L^{n} f\right)(b)=0,0 \leqslant n<k\right\}$.

The classes $A_{\nu}\{L ;\langle a, b\rangle\}$ are obtained from $B_{\nu}^{(\infty)}\{L ;\langle a, b\rangle\}$ by putting on the requirement $f \in A\langle a, b\rangle$ over and above $f \in C^{(\infty)}\langle a, b\rangle$.

The functions $f(x)$ we consider are ones that satisfy $\int_{a}^{b} P(x)\left|\left(L^{n} f\right)(x)\right|^{2} d x<\infty$ $(n=0,1,2, \ldots)$ and which are members of some $A_{\nu}$. In other words, all of the successive iterates $f(x),(L f)(x),\left(L^{2} f\right)(x), \ldots$ are in the weighted Lebesgue space $L^{2}(P ;(a, b))$ and satisfy the boundary conditions pertaining to the $A_{\nu}$ which contains $f(x)$.

The eigenfunctions $u_{n}(x)$ in (1.4) will belong to the same $A_{\nu}$ as $f(x)$ does. In cases $\nu=1,2$ the boundary conditions $f(a)=f(b)=0$ and $f(a)=f(b), f^{\prime}(a)=f^{\prime}(b)$, are the standard ones used to define the domain of a selfadjoint Hilbert space operator. In our case, the operator $\mathrm{T}_{\nu}$ is the restriction of $\tilde{L}$ to $B_{\nu}^{(1)}\{L ;[a, b]\}$ for $\nu=1$ or 2 . Therefore, the eigenvalues $\left\{\mu_{n}\right\}$ are just the usual ones comprising the point spectrum of $\mathrm{T}_{\nu}$, and the expansion (1.4) comes about by taking an operator with a discrete spectrum. However, the condition $f(x) \in A_{\nu}[a, b]$ does not say that all the iterates $\left(L^{n} f\right)(x)$ belong to the domain of $\mathrm{T}_{\nu}$ unless $L=\tilde{L}$ (i.e. $L$ is symmetric, or "formally selfadjoint") simply because $(\tilde{L} f)(x)=P(x)(L f)(x) \neq$ $(L f)(x)$. 
In cases $\nu=3,4$ the boundary conditions are not the usual ones encountered in spectral theory. However if $L=\tilde{L}$, we may introduce selfadjoint Hilbert space operators $\mathrm{T}_{\nu}$ whose eigenfunctions do satisfy the conditions of $A_{\nu}\langle a, b\rangle$. Such a calculation is done in [15] for the case $\nu=4$ with $x=a$ regular and $x=b$ singular. The boundary condition involves a certain function $\chi(t)$, which is a solution to (1.1) with $\lambda=0$ and which belongs to $L^{2}[a, b)$. The boundary condition at $x=b$ given by

$$
B^{*}(f)=\lim _{x \rightarrow b}\left[p_{2}(x)\left(\chi(x) f^{\prime}(x)-\chi^{\prime}(x) f(x)\right)\right]=0
$$

coupled with a constraint $c_{1} f(a)+c_{2} f^{\prime}(a)=0$, give rise to a selfadjoint operator $[4,20,6]$. It is proved in [15, Lemma 2.4], that members $f(x)$ of the domain of the operator always satisfy $\lim _{x \rightarrow b}[f(x) / y(x, a ; \lambda)]=0$, at least for $\lambda>0$ sufficiently large. In many cases it is permissable to take $\lambda>0$ arbitrarily small, and here the condition coincides exactly with that of $B_{4}^{(1)}\{L ;[a, b)\}$. Consequently, the eigenvalues and eigenfunctions are realizable as those of a conventional Hilbert space operator.

If we are dealing with a nonsymmetric $L$, the Laguerre operator for instance, then we are forced to rely on Hille's axiomatic approach. That is, we will assume the existence of a complete orthonormal set of eigenfunctions $\left\{u_{n}(x)\right\}_{n=1}^{\infty}$, each of which satisfies the boundary conditions of the appropriate class $A_{\nu}$.

The reason the classes $A_{\nu}$ are used is that they expediate Hille's work on the "oscillation preserving" nature of operators of type $\mathrm{T}_{\nu}$. Given a function $g \in$ $C^{(0)}(a, b)$, we let $V[g]$ denote the number of sign changes of $g(x)$ in $(a, b)$. To say that $g(x)$ has $N$ sign changes in $(a, b)$ will mean that $(a, b)$ breaks up into exactly $N+1$ subintervals in each of which $g(x)$ keeps a constant sign, the signs being opposite in adjacent intervals [5, p. 466]. In the periodic case we identify the endpoints $x=a$ and $x=b$, viewing $g(x)$ as being defined on the circle. Here $N$ intervals determine $N$ sign changes.

Let $F$ be a subclass of $C^{(2)}(a, b)$, and let $\lambda$ be fixed and real. Hille [5, p. 468] terms the operator $L-\lambda$ oscillation preserving in $(a, b)$ with respect to $F$ if

$$
V[(L-\lambda) f(x)] \geqslant V[f(x)] \text { for every } f \in F .
$$

The following result is crucial for our purposes.

THEOREM C [5, p. 472]. If the operator $L$ is of type $\mathrm{T}_{\nu}$ and $\lambda>0$, then $L-\lambda$ is oscillation preserving with respect to the corresponding class $B_{\nu}^{(1)}\{L ;\langle a, b\rangle\}$.

We remark that for type $\mathrm{T}_{1}$, the condition $f(a)=0$ may be replaced by $c_{1} f(a)-$ $c_{2} f^{\prime}(a)=0$, where $c_{1} \geqslant 0$ and $c_{2}>0$, if $f(b)=0$ is left unchanged [5, pp. 472-473] and this does not affect the truth of Theorem $C$. The roles of $a$ and $b$ may be interchanged. Also, the case $f^{\prime}(a)=0, f^{\prime}(b)=0$ leads to an altered class $B_{1}^{(1)}\{L ;[a, b]\}$ with respect to which $L-\lambda$ is still oscillation preserving.

Consider now a system $S=S\left\{L, u_{n}(x), \mu_{n} ;(a, b)\right\}$ consisting of the operator $L$, a set of eigenfunctions $u_{n}(x)$, and eigenvalues $\mu_{n}$ on the interval $(a, b)$. We say that $S$ 
is admissible [5, p. 478] if $L$ is of one of the four types $T_{\nu}$ and, in addition to axioms $\left(A_{1}\right)$ and $\left(A_{2}\right)$, the following axioms $\left(A_{3}\right)-\left(A_{6}\right)$ hold.

$\left(\mathrm{A}_{3}\right)$ The functions $\left\{[P(x)]^{1 / 2} u_{n}(x)\right\}$ form a real, complete orthonormal system in $L^{2}(P ;(a, b))$.

Of course the eigenfunctions satisfy the boundary conditions pertaining to $T_{\nu}$. Written out, the alternative boundary problems are:

$\left(\mathrm{P}_{1}\right)(L+\mu) u=0, u(a)=u(b)=0$;

$\left(\mathrm{P}_{2}\right)(L+\mu) u=0, u(a)=u(b), u^{\prime}(a)=u^{\prime}(b)$;

$\left(\mathrm{P}_{3}\right)(L+\mu) u=0,\left[u(x) / y\left(x ; x_{0}, \lambda\right)\right] \rightarrow 0, x \rightarrow a, b$;

$\left(\mathrm{P}_{4}\right)(L+\mu) u=0,[u(x) / y(x ; b, \lambda)] \rightarrow 0, x \rightarrow a, c_{1} u(b)+c_{2} u^{\prime}(b)=0$.

$\left(\mathrm{A}_{4}\right) 0<\mu_{n} \leqslant \mu_{n+1} ; \sum_{n=1}^{\infty} \mu_{n}^{-\alpha}<\infty$ for some $\alpha>0$.

$\left(\mathrm{A}_{5}\right)$ There exist constants $\beta$ and $\gamma$, and a nonnegative function $U(x) \in C^{(0)}(a, b)$ such that $\left|u_{n}(x)\right| \leqslant \mu_{n}^{\beta} U(x),\left|u_{n}^{\prime}(x)\right| \leqslant \mu_{n}^{\gamma} U(x)$.

$\left(\mathrm{A}_{6}\right) V\left[u_{n}(x)\right]=n$ unless $u_{n}(x)$ corresponds to a double eigenvalue, and then $V\left[u_{n}(x)\right] \geqslant n-1$.

Our axiom $\left(\mathrm{A}_{6}\right)$ differs from that of [5], because we are seeking a stronger result. In the periodic case the first eigenfunction may be zero-free. We prove our main results below under the assumption $V\left[u_{n}(x)\right]=n$; at the end of $\S 4$, we indicate how the proofs can be modified to cover double eigenvalues. In $\left(\mathrm{A}_{4}\right)$ we have reflected the possibility of double eigenvalues by writing $\mu_{n} \leqslant \mu_{n+1}$.

A set $F=F\left\{L, u_{n}(x), \mu_{n} ;(a, b)\right\}$ of eigenfunction series $f(x)=\sum_{n=1}^{\infty} f_{n} u_{n}(x)$ will be termed admissible if $S$ is admissible and

$\left(\mathrm{C}_{1}\right) f_{n}$ is real for each $n$,

$\left(\mathrm{C}_{2}\right) \sum_{n=1}^{\infty} \mu_{n}^{k}\left|f_{n}\right|<\infty, k=0,1,2, \ldots$

An individual member $f(x) \in F$ is called an admissible function. As Hille notes [5, pp. 479-481] these are functions $f(x)$ for which (1.4) and (1.5) hold, with uniform convergence on compact sets, and moreover, $\int_{a}^{b} P(x)\left|\left(L^{n} f\right)(x)\right|^{2} d x<\infty$ for all $n$ and $f_{n}=\int_{a}^{b} P(t) u_{n}(t) f(t) d t$. In cases $\nu=1$ or $2, F=B_{\nu}^{(\infty)}$, while $F \subset B_{\nu}^{(\infty)}$ in the other cases.

Hille's final axioms are:

$\left(\mathrm{D}_{\nu}\right)$ If $L$ is of type $\mathrm{T}_{\nu}$ then $u_{n}(x) \in A_{\nu}\{L ;\langle a, b\rangle\}$ for all $n$. Thus the eigenfunctions satisfy $\left(\mathrm{P}_{\nu}\right)$.

$\left(\mathrm{E}_{3}\right)$ If $\nu=3$ there exists a finite constant $C\left(x_{0} ; \lambda\right)$ such that $U(x) \leqslant$ $C\left(x_{0}, \lambda\right) y\left(x ; x_{0}, \lambda\right), a<x<b, \lambda>0$.

$\left(\mathrm{E}_{4}\right)$ If $\nu=4$, there exists a finite constant $C(\lambda)$ such that $U(x) \leqslant C(\lambda) y(x ; b, \lambda)$, $a<x<b, \lambda>0$.

An admissible system $S$ which satisfies the conditions $\left(\mathrm{D}_{\nu}\right)$ and $\left(\mathrm{E}_{\nu}\right)$ corresponding to its type $\mathrm{T}_{\nu}$ is called conservative.

This completes our discussion of the axioms of [5]. We require one more assumption. We will suppose that there is a sequence $\left\{\alpha_{n}\right\}_{n=1}^{\infty}$ and a constant $\delta>0$ such that $1 \leqslant\left(1 / \alpha_{n}\right) \leqslant \mu_{n}^{\delta}$ for all $n$, and

$\left(\mathrm{A}_{7}\right)\left|u_{n}(x) /\left(\alpha_{n} U(x)\right)\right|$ attains a value between each pair of consecutive zeros of $u_{n}(x)$, and on either side of the interval of oscillation of $u_{n}(x)$, of at least 1 . If $\mu_{n}$ is a double eigenvalue with eigenfunctions $u_{n}(x)$ and $\tilde{u}_{n}(x)$ then $\left|\left[u_{n}(x)+\sigma \tilde{u}_{n}(x)\right] /\left(\alpha_{n} U(x)\right)\right|$ attains such values for every real parameter $\sigma$. 
For regular Sturm-Liouville problems with separated endpoint conditions, the $\alpha_{n}$ may be taken as constants. The assumption on double eigenvalues in $\left(A_{7}\right)$ is patterned after the periodic case, and specifically the condition that $\mid \sin (2 m \pi x)+$ $\sigma \cos (2 m \pi x) \mid$ has extrema of $\left(1+\sigma^{2}\right)^{1 / 2}$ between its consecutive zeros. For the classical orthogonal polynomials $\alpha_{n}^{-1}$ has order of magnitude $n^{1 / 2}$ for Legendre and Jacobi, and $n^{1 / 4}$ for Hermite and Laguerre [1].

Supposing the eigenvalues $\mu_{n}$ to be simple, let us define the positive quantities

$$
\theta_{m}=\mu_{m+1}-\mu_{m}, \quad \omega_{m}=\mu_{m+1}^{1 / 2}-\mu_{m}^{1 / 2}, \quad m=1,2,3, \ldots
$$

The objectives of $\S \S 3$ and 4 are to prove the following two theorems for the case of simple eigenvalues. In later remarks, we indicate how to modify them to allow double eigenvalues.

THEOREM 1. Let $f(x)$ be admissible and satisfy

$$
\limsup _{n \rightarrow \infty}\left|f_{n}\right| e^{\tau \mu_{n}^{1 / 2}}=\infty
$$

for some $\tau>0$. Let $\left\{c_{m}\right\}_{m=1}^{\infty}$ be a sequence such that $k_{m}=c_{m} \mu_{m}^{2}$ is an integer for each $m$, and for all $m$ sufficiently large,

$$
\begin{gathered}
c_{m} \mu_{m} \geqslant 1, \\
\theta_{m} / \mu_{m} \leqslant 1, \\
0<\theta_{1}<\theta_{2}<\cdots, \\
c_{m} \theta_{m-1} \geqslant \tau(3+2 \tau), \\
c_{m} \mu_{m}^{2} \geqslant 2(\beta+\delta) \mu_{m+1} / \theta_{1}, \\
m \mu_{m+1}^{\beta+\delta+1} \exp \left[\theta_{m-1}\left(\tau-c_{m} \theta_{m-1} / 9\right)\right] \rightarrow 0, \quad m \rightarrow \infty .
\end{gathered}
$$

Then for infinitely many $m, V\left[\left(L^{k_{m}} f\right)(x)\right] \geqslant m$.

THEOREM 2. Let $f(x)$ be admissible and satisfy (2.3) for some $\tau>0$. Let $\left\{d_{m}\right\}_{m=1}^{\infty}$ be a sequence such that for all $m, \tilde{k}_{m}=d_{m} \mu_{m}$ is an integer, and for large $m$,

$$
\begin{gathered}
\omega_{m} \geqslant K>0 \quad \text { and }\left(\omega_{m} / \mu_{m}^{1 / 2}\right) \leqslant 1 \quad(K \text { constant }), \\
d_{m} \mu_{m}^{1 / 2} \geqslant \max \left\{\frac{(\beta+\gamma) \mu_{m+1}}{K \mu_{m}}, \frac{\tau+1}{\ln 4}\right\}, \\
m \mu_{m+1}^{\beta+\delta+1} \exp \left[-d_{m} \omega_{m-j}^{i+1} / 3\right] \rightarrow 0, \quad m \rightarrow \infty, \text { for } i, j=0,1 .
\end{gathered}
$$

Then for infinitely many $m, V\left[\left(L^{\tilde{k_{m}}} f\right)(x)\right] \geqslant m$.

3. Proof of Theorem 1. Following Pólya and Wiener, we start off with the following preliminary fact [10, Lemma II, p. 252].

LEMMA. Let $\left\{l_{n}\right\}_{1}^{\infty}$ and $\left\{s_{n}\right\}_{1}^{\infty}$ be two sequences such that $l_{n} \geqslant 0,0<s_{1}<s_{2}<\cdots$, $\lim _{n \rightarrow \infty} l_{n}=0$ and $\lim \sup _{n \rightarrow \infty} l_{n} s_{n}=\infty$. Then there exists an infinite set of integers $\{m\}$ such that $l_{m} \geqslant l_{n}, n \geqslant m$, and $l_{m} s_{m} \geqslant l_{n} s_{n}, n \leqslant m$. 
For the proofs of Theorems 1 and 2 we will put $f_{n}^{*}=\alpha_{n} f_{n}$ and $u_{n}^{*}(x)=\alpha_{n}^{-1} u_{n}(x)$, so that $f_{n} u_{n}(x)=f_{n}^{*} u_{n}^{*}(x)$. Now suppose (2.3) holds. Then

$$
\left|f_{n}\right| e^{\tau \mu_{n}^{1 / 2}}=\left|f_{n}^{*}\right| \alpha_{n}^{-1} e^{\tau \mu_{n}^{1 / 2}} \leqslant\left|f_{n}^{*}\right| \mu_{n}^{\delta} e^{\tau \mu_{n}^{1 / 2}} \leqslant\left|f_{n}^{*}\right| e^{\tau \mu_{n}}
$$

for $n$ sufficiently large. Thus $\lim \sup _{n \rightarrow \infty}\left|f_{n}^{*}\right| e^{\tau \mu_{n}}=\infty$. Setting $l_{n}=\left|f_{n}^{*}\right|$ and $s_{n}=e^{\tau \mu_{n}}$, $\left(\mathrm{C}_{2}\right)$ implies $l_{n} \rightarrow 0$. The lemma then yields an infinite set $\Re$ of integers so that

$$
\left|f_{m}^{*}\right| \geqslant\left|f_{n}^{*}\right|, \quad n \geqslant m, m \in \mathfrak{N} ; \quad\left|f_{m}^{*}\right| e^{\tau \mu_{m}} \geqslant\left|f_{n}^{*}\right| e^{\tau \mu_{n}}, \quad n \leqslant m, m \in \mathfrak{N} .
$$

As in [5], we introduce the function

$$
\Phi_{m, k}(x ; f)=\sum_{n=1}^{\infty}\left\{\frac{4 \mu_{m} \mu_{n}}{\left(\mu_{m}+\mu_{n}\right)^{2}}\right\}^{k} f_{n} u_{n}(x)
$$

for each pair $m, k$ of positive integers. Then, by repeatedly applying the operator $\left(L-\mu_{m}\right)$ to $\Phi_{m, k}$, and noting $L u_{n}(x)=-\mu_{n} u_{n}(x)$, we have

$$
\left(L-\mu_{m}\right)^{2 k} \Phi_{m, k}(x ; f)=\left(-4 \mu_{m}\right)^{k}\left(L^{k} f\right)(x) .
$$

Then by Theorem C,

$$
V\left[\left(L^{k} f\right)(x)\right] \geqslant V\left[\Phi_{m, k}(x ; f)\right] .
$$

Our aim is to prove $V\left[\Phi_{m, k}(x ; f)\right] \geqslant m$, where $k=k_{m}$.

Recalling $f_{n} u_{n}(x)=f_{n}^{*} u_{n}^{*}(x), f_{n}^{*}=\alpha_{n} f_{n}, u_{n}^{*}(x)=\alpha_{n}^{-1} u_{n}(x)$, write

$$
\begin{aligned}
\Phi_{m, k}(x ; f) & =f_{m}^{*} u_{m}^{*}(x)+S_{1}(x)+S_{2}(x), \\
S_{1}(x) & =\sum_{n=1}^{m-1}\left\{\frac{4 \mu_{m} \mu_{n}}{\left(\mu_{m}+\mu_{n}\right)^{2}}\right\}^{k} f_{n}^{*} u_{n}^{*}(x), \\
S_{2}(x) & =\sum_{n=m+1}^{\infty}\left\{\frac{4 \mu_{m} \mu_{n}}{\left(\mu_{m}+\mu_{n}\right)^{2}}\right\}^{k} f_{n}^{*} u_{n}^{*}(x),
\end{aligned}
$$

so that

$$
\frac{\Phi_{m, k}(x ; f)}{f_{m}^{*} U(x)}=\frac{u_{m}^{*}(x)}{U(x)}+\frac{S_{1}(x)}{f_{m}^{*} U(x)}+\frac{S_{2}(x)}{f_{m}^{*} U(x)} .
$$

By $\left(\mathrm{A}_{7}\right)$, the first term on the right of (3.3) has $m$ zeros exactly in $(a, b)$, and its absolute value attains the value 1 at least once between consecutive zeros and on each side of the interval of oscillation. We are going to show that the terms $\left|S_{i}(x) /\left[f_{m}^{*} U(x)\right]\right|$ are uniformly small over $(a, b)$, for $m$ sufficiently large and $k=k_{m}$. Hence, the left side of (3.3) will have at least $m$ sign changes in $(a, b)$, and, in view of (3.2), we will have the desired conclusion. 
To this end, consider first $S_{2}(x) / f_{m}^{*} U(x)$ for $m \in \mathfrak{N}$. Using (3.1), $\left(\mathrm{A}_{5}\right)$ and the definition of $\left\{\alpha_{n}\right\}$ we have

$$
\begin{aligned}
\left|\frac{S_{2}(x)}{f_{m}^{*} U(x)}\right| & \leqslant \sum_{n=m+1}^{\infty}\left\{\frac{4 \mu_{n} \mu_{m}}{\left(\mu_{n}+\mu_{m}\right)^{2}}\right\}^{k}\left|\frac{f_{n}^{*}}{f_{m}^{*}} \frac{u_{n}(x)}{\alpha_{n} U(x)}\right| \\
& \leqslant \sum_{n=m+1}^{\infty}\left\{\frac{4 \mu_{n} \mu_{m}}{\left(\mu_{n}+\mu_{m}\right)^{2}}\right\}^{k} \mu_{n}^{\beta+\delta} \\
& \leqslant \mu_{m}^{\beta+\delta} \sum_{n=m+1}^{\infty}\left\{\frac{4\left(\mu_{n} / \mu_{m}\right)}{\left(1+\left(\mu_{n} / \mu_{m}\right)\right)^{2}}\right\}^{k}\left(\mu_{n} / \mu_{m}\right)^{\beta+\delta} .
\end{aligned}
$$

Let $h=\beta+\delta$, and define $g(x)=\left[4 x /(1+x)^{2}\right]^{k} x^{h}$. Then the right of (3.4) is the same as

$$
\mu_{m}^{h}\left[g\left(\mu_{m+1} / \mu_{m}\right)+g\left(\mu_{m+2} / \mu_{m}\right)+\cdots\right]
$$

The function $g(x)$ takes its absolute maximum at the point $x_{\max }=(k+h) /(k-h)$. Now $x_{\max }<\left(\mu_{m+1} / \mu_{m}\right)$, for this is equivalent to

$$
\begin{aligned}
(k+h) /(k-h) & <\left(\mu_{m+1} / \mu_{m}\right) \Leftrightarrow k \mu_{m}+h \mu_{m}<(k-h)\left(\mu_{m}+\theta_{m}\right) \\
& \Leftrightarrow h\left(\theta_{m}+2 \mu_{m}\right)<k \theta_{m} \Leftrightarrow h\left(\mu_{m}+\mu_{m+1}\right)<k \theta_{m}
\end{aligned}
$$

which is implied by $2 h \mu_{m+1}<k \theta_{1} \Leftrightarrow c_{m} \mu_{m}^{2}>\left(2 h / \theta_{1}\right) \mu_{m+1}$ where $k=k_{m}=c_{m} \mu_{m}^{2}$. However, this last condition is (2.8), and hence, $x_{\max }<\left(\mu_{m+1} / \mu_{m}\right)$. Consequently, $g(x)$ decreases over $\left(\left(\mu_{m+1} / \mu_{m}\right), \infty\right)$. Thus, (3.5) can be replaced by

$$
\begin{aligned}
& \mu_{m}^{h}\left\{g\left(\mu_{m+1} / \mu_{m}\right)+\left[g\left(\mu_{m+2} / \mu_{m}\right) \frac{\left(\theta_{m+1} / \mu_{m}\right)}{\left(\theta_{m+1} / \mu_{m}\right)}\right.\right. \\
& \left.\left.+g\left(\mu_{m+3} / \mu_{m}\right) \frac{\left(\theta_{m+2} / \mu_{m}\right)}{\left(\theta_{m+2} / \mu_{m}\right)}+\cdots\right]\right\} \\
& \leqslant \mu_{m}^{h} g\left(\mu_{m+1} / \mu_{m}\right)+\mu_{m}^{1+h} \theta_{1}^{-1} \int_{\left(\mu_{m+1} / \mu_{m}\right)}^{\infty} g(x) d x
\end{aligned}
$$

The first term on the right of (3.6) is

$$
\begin{aligned}
\mu_{m}^{h} g\left(\mu_{m+1} / \mu_{m}\right) & =\mu_{m+1}^{h}\left[\frac{4 \mu_{m+1} \mu_{m}}{\left(\mu_{m+1}+\mu_{m}\right)^{2}}\right]^{k}=\mu_{m+1}^{h}\left[\frac{4 \mu_{m}\left(\mu_{m}+\theta_{m}\right)}{\left(2 \mu_{m}+\theta_{m}\right)^{2}}\right]^{k} \\
& =\mu_{m+1}^{h}\left[1-\frac{1}{\left(2\left(\mu_{m} / \theta_{m}\right)+1\right)^{2}}\right]^{k}
\end{aligned}
$$


Substituting $E_{m}=2\left(\mu_{m} / \theta_{m}\right)+1$, this may be expressed as

$$
\begin{aligned}
\mu_{m+1}^{k}\left(1-1 / E_{m}^{2}\right)^{k} & =\mu_{m+1}^{h}\left\{\left(1-1 / E_{m}^{2}\right)^{E_{m}^{2}}\right\}^{\left(k / E_{m}^{2}\right)} \\
& <\mu_{m+1}^{h}(1 / e)^{\left(k / E_{m}^{2}\right)}=\mu_{m+1}^{h} \exp \left\{-\left[c_{m} \mu_{m}^{2} /\left(2\left(\mu_{m} / \theta_{m}\right)+1\right)^{2}\right]\right\} \\
& =\mu_{m+1}^{h} \exp \left[-c_{m} \theta_{m}^{2} /\left[2+\left(\theta_{m} / \mu_{m}\right)\right]^{2}\right] \\
& <\mu_{m+1}^{h} \exp \left[-c_{m} \theta_{m}^{2} / 9\right] \quad(\text { by }(2.5)) \\
& <e^{-\tau \theta_{1}} m \mu_{m+1}^{h} e^{\tau \theta_{m-1}} \exp \left[-c_{m} \theta_{m} / 9\right] \quad(\text { by }(2.6)) \\
& =e^{-\tau \theta_{1}} m \mu_{m+1}^{\beta+\delta} \exp \left(\theta_{m-1}\right)\left[\tau-\left(c_{m} \theta_{m-1} / 9\right)\left(\theta_{m} / \theta_{m-1}\right)^{2}\right] \\
& <e^{-\tau \theta_{1}} m \mu_{m+1}^{\beta+\delta} \exp \left(\theta_{m-1}\right)\left[\tau-c_{m} \theta_{m-1} / 9\right] \quad(\text { by }(2.6))
\end{aligned}
$$

which converges to 0 by (2.9).

The other term in (3.6) is the integral $\int_{\left(\mu_{m+1} / \mu_{m}\right)}^{\infty} g(x) d x$ in which $k=k_{m}$. Break the integral into parts ranging from $\left(\mu_{m+1} / \mu_{m}\right)$ to $X$, and from $X$ to $\infty$, where $X$ is to be chosen. We have

$$
\int_{X}^{\infty}\left[\frac{4 x}{(1+x)^{2}}\right]^{k} x^{h} d x<\int_{X}^{\infty} 4^{k}(1+x)^{-k+h} d x=\frac{(1+X)^{1+h}}{k-h-1}\left(\frac{4}{1+X}\right)^{k},
$$

when $k=k_{m}$ is large enough. The factor multiplying this integral is $\mu_{m}^{1+h} \theta_{1}^{-1}$, but $k_{m}=c_{m} \mu_{m}^{2}$, so $X>3$ implies that the truncated integral on the right of (3.6) does not exceed

$$
\frac{\mu_{m}^{1+h}}{\theta_{1}}\left(\frac{4}{1+X}\right)^{c_{m} \mu_{m}^{2}} \frac{(1+X)^{1+h}}{k-h-1} \rightarrow 0 \quad \text { as } m \rightarrow \infty .
$$

As for the range $\left(\mu_{m+1} / \mu_{m}\right) \leqslant x \leqslant X$, we replace the integral by

$$
\int_{\left(\mu_{m+1} / \mu_{m}\right)}^{X} g(x) d x \leqslant g\left(\mu_{m+1} / \mu_{m}\right) X
$$

if $\left(\mu_{m+1} / \mu_{m}\right) \leqslant X$. Multiplying by the factor $\mu_{m}^{1+h} \theta_{1}^{-1}$, we get

$$
\frac{\mu^{1+h}}{\theta_{1}} \int_{\left(\mu_{m+1} / \mu_{m}\right)}^{X} g(x) d x<\frac{X}{\theta_{1}} \mu_{m}^{1+h} g\left(\mu_{m+1} / \mu_{m}\right)
$$

and this converges to zero by the above argument. Taken together, we have now proved $\left|S_{2}(x) / f_{m}^{*} U(x)\right| \rightarrow 0$ uniformly on $(a, b)$ as $m \rightarrow \infty, m \in \mathfrak{T}$.

As for the other sum in (3.3), we use (3.1), $\left(\mathrm{A}_{5}\right)$ and the definition of $\alpha_{n}$ again to estimate it by

$$
\left|\frac{S_{1}(x)}{f_{n}^{*} U(x)}\right| \leqslant \sum_{n=1}^{m-1}\left\{\frac{4 \mu_{m} \mu_{n}}{\left(\mu_{m}+\mu_{n}\right)^{2}}\right\}^{k} \mu_{n}^{\beta+\delta} e^{\tau\left(\mu_{m}-\mu_{n}\right)} .
$$

We consider the expression $\left[4 x_{0} x /\left(x_{0}+x\right)^{2}\right]^{k} e^{\tau\left(x_{0}-x\right)}$; we will eventually set $x_{0}=$ $\mu_{m}$ and $x=\mu_{n}$. After a calculation, one sees that the expression has derivative, with 
respect to $x$, which is a strictly negative multiple of $\left[\tau x^{2}+\left(\tau x_{0}+k\right) x-k x_{0}\right]$, and so the derivative vanishes precisely at the points

$$
x_{( \pm)}=\left[-\left(\tau x_{0}+k\right) \pm \sqrt{\left(\tau x_{0}+k\right)^{2}+4 k x_{0} \tau}\right] /(2 \tau) .
$$

Disregarding the negative root, we compute that

$$
x_{(+)}=\frac{2 k x_{0} /\left(\tau x_{0}+k\right)}{\left[1+\left(4 \tau k x_{0} /\left(\tau x_{0}+k\right)^{2}\right)\right]^{1 / 2}+1} .
$$

Clearly $x_{(+)} \geqslant \tilde{x}_{(+)}$, where

$$
\tilde{x}_{(+)}=\frac{k x_{0} /\left(\tau x_{0}+k\right)}{1+\left[2 \tau k x_{0} /\left(\tau x_{0}+k\right)^{2}\right]} .
$$

With $x_{0}=\mu_{m}$ and $x=\mu_{n}$ we claim $\tilde{x}_{(+)} \geqslant \mu_{m-1}$, for this is equivalent to

$$
\begin{aligned}
\frac{k \mu_{m}}{\tau \mu_{m}+k} & \geqslant \mu_{m-1}\left(1+\frac{2 \tau k \mu_{m}}{\left(\tau \mu_{m}+k\right)^{2}}\right) \\
& \Leftrightarrow \frac{\mu_{m}}{\mu_{m-1}} \geqslant\left(1+\frac{\tau \mu_{m}}{k}\right)\left(1+\frac{2 \tau k \mu_{m}}{\left(\tau \mu_{m}+k\right)^{2}}\right) \\
& \Leftrightarrow \frac{\mu_{m-1}+\theta_{m-1}}{\mu_{m-1}} \geqslant\left(1+\frac{\tau \mu_{m}}{k}\right)\left(1+\frac{2 \tau \mu_{m}}{k\left(1+\left(\tau \mu_{m} / k\right)\right)^{2}}\right) .
\end{aligned}
$$

This is implied by

$$
\begin{aligned}
\left(1+\frac{\theta_{m-1}}{\mu_{m-1}}\right) & \geqslant\left(1+\frac{\tau \mu_{m}}{k}\right)\left(1+\frac{2 \tau \mu_{m}}{k}\right) \\
& \Leftrightarrow\left(1+\frac{\theta_{m-1}}{\mu_{m-1}}\right) \geqslant 1+\frac{3 \tau \mu_{m}}{k}+\frac{2 \tau^{2} \mu_{m}^{2}}{k^{2}} \\
& \Leftrightarrow \frac{\theta_{m-1}}{\mu_{m-1}} \geqslant 1+\frac{3 \tau}{c_{m} \mu_{m}}+\frac{2 \tau^{2}}{c_{m}^{2} \mu_{m}^{2}} \quad\left(k=k_{m}=c_{m} \mu_{m}^{2}\right),
\end{aligned}
$$

which is implied by

$$
\begin{aligned}
\frac{\theta_{m-1}}{\mu_{m-1}} & \left.\geqslant \frac{3 \tau}{c_{m} \mu_{m}}+\frac{2 \tau^{2}}{c_{m} \mu_{m}} \quad \text { (by }(2.4)\right) \\
& \Leftrightarrow c_{m} \theta_{m-1} \geqslant \tau(3+2 \tau)\left(\mu_{m-1} / \mu_{m}\right),
\end{aligned}
$$

which is implied by (2.7) since $\mu_{m-1}<\mu_{m}$. Consequently, $x_{(+)} \geqslant \mu_{m-1}$, and this says that the expression $\left[4 \mu_{m} \mu_{n} /\left(\mu_{m}+\mu_{n}\right)^{2}\right]^{k} \exp \left(\tau \mu_{m}-\tau \mu_{n}\right)$ is maximized by taking 
$n=m-1$. Therefore,

$$
\begin{aligned}
\left|\frac{S_{1}(x)}{f_{m}^{*} U(x)}\right| & \leqslant(m-1) \mu_{m-1}^{\beta+\delta}\left[\frac{4 \mu_{m} \mu_{m-1}}{\left(\mu_{m}+\mu_{m-1}\right)^{2}}\right]^{k} e^{\tau\left(\mu_{m}-\mu_{m-1}\right)} \\
& =(m-1) \mu_{m-1}^{\beta+\delta}\left[\frac{4 \mu_{m-1}\left(\mu_{m-1}+\theta_{m-1}\right)}{\left(2 \mu_{m-1}+\theta_{m-1}\right)^{2}}\right]^{k} e^{\tau \theta_{m-1}} \\
& =(m-1) \mu_{m-1}^{\beta+\delta}\left(1-\frac{1}{E_{m-1}^{2}}\right)^{k} e^{\tau \theta_{m-1}}
\end{aligned}
$$

where $E_{m-1}=2\left(\mu_{m-1} / \theta_{m-1}\right)+1$. Hence, we have

$$
\begin{aligned}
\left|\frac{S_{1}(x)}{f_{n}^{*} U(x)}\right| & \leqslant(m-1) \mu_{m-1}^{\beta+\delta}(1 / e)^{\left(k / E_{m-1}^{2}\right)} e^{\tau \theta_{m-1}} \\
& =(m-1) \mu_{m-1}^{\beta+\delta} \exp \left[\tau \theta_{m-1}-\frac{k \theta_{m-1}^{2}}{\left(2 \mu_{m-1}+\theta_{m-1}\right)^{2}}\right] \\
& =(m-1) \mu_{m-1}^{\beta+\delta} \exp \theta_{m-1}\left[\tau-\frac{c_{m} \theta_{m-1}}{\left[2+\left(\theta_{m-1} / \mu_{m-1}\right)\right]^{2}}\right] \quad\left(k=c_{m} \mu_{m}^{2}\right) \\
& \leqslant(m-1) \mu_{m-1}^{\beta+\delta} \exp \theta_{m-1}\left(\tau-\frac{c_{m} \theta_{m-1}}{9}\right) \quad(\text { by }(2.5)),
\end{aligned}
$$

and this approaches 0 as $m \rightarrow \infty$ by (2.9).

In view of the remarks below (3.3), the proof of Theorem 1 is complete.

Corollary 1. Assume that the eigenvalues have the asymptotic form

$$
\mu_{n}=\alpha n^{p}(1+o(1)), \quad n \rightarrow \infty,
$$

where $p \geqslant 1$ and $\alpha$ is constant, and specifically $\mu_{n}=\alpha n^{p}\left(1+\varepsilon_{n}\right)$ where $((n+1) / n)^{p}$ $\geqslant \varepsilon_{n} / \varepsilon_{n+1} \geqslant 1$, for all $n$, and $\varepsilon_{n} \rightarrow 0$. Let $f(x)$ be admissible and suppose that for each $\varepsilon>0$ there is a constant $A>0$ such that $V\left[\left(L^{k} f\right)(x)\right] \leqslant A k^{1 /(1+p+\varepsilon)}$ for all $k$ sufficiently large. Then $\lim \sup \left|f_{n}\right| e^{\tau \mu_{n}^{1 / 2}}<\infty$ for all $\tau>0$.

Proof. By our assumption, $\theta_{n} \geqslant \alpha p n^{p-1}$. If $c_{n}$ is selected so that $c_{n}>n^{1-p+\varepsilon_{1}}$, where $0<\varepsilon_{1}<\varepsilon$, then (2.4)-(2.9) are easily seen to hold. In fact, we choose $c_{n}$ so that

$$
n^{1-p+\varepsilon_{1}}<c_{n}<\frac{n^{1-p+\varepsilon}}{\alpha^{2}\left(1+\varepsilon_{n}\right)^{2} A^{1+p+\varepsilon}}
$$

and put $k_{n}=c_{n} \mu_{n}^{2}=c_{n} \alpha^{2} n^{2 p}\left(1+\varepsilon_{n}\right)^{2}$. Since this imposes only

$$
\alpha^{2} n^{1+p+\varepsilon_{1}}\left(1+\varepsilon_{n}\right)^{2}<k_{n}<(n / A)^{1+p+\varepsilon}
$$


we see that there are choices of $c_{n}$ which make $k_{n}$ an integer. Observe that

$$
\begin{aligned}
V\left[\left(L^{k_{n}} f\right)(x)\right] & \leqslant A\left(k_{n}\right)^{1 /(1+p+\varepsilon)}=A\left[c_{n} \alpha^{2} n^{2 p}\left(1+\varepsilon_{n}\right)^{2}\right]^{1 /(1+p+\varepsilon)} \\
& <A\left[\frac{n^{1-p+\varepsilon} n^{2 p}}{A^{1+p+\varepsilon}}\right]^{1 /(1+p+\varepsilon)}=n,
\end{aligned}
$$

and this contradicts Theorem 1 unless (2.3) fails. The completes the proof.

When $p=1$ we can thus come to within $\varepsilon$ of $k^{1 / 2}$ for the upper limit of $V\left[\left(L^{k} f\right)(x)\right]$. Hermite and Laguerre expansions are covered by this situation. For $p=2$, however, the corollary only gives (to within $\varepsilon$ of) $k^{1 / 3}$ which, in view of Hille's Theorem B, is not the right order for Legendre and Jacobi expansions. We shall see that $k^{1 / 2}$ (within $\varepsilon$ ) is obtainable for these expansions through Theorem 2.

4. Proof of Theorem 2. Noting (2.3), set $l_{n}=\left|f_{n}\right|$ and $s_{n}=e^{\tau \mu_{n}^{1 / 2}}$, and apply the lemma to obtain an infinite set $\Re$ of integers such that

$$
\begin{gathered}
\left|f_{m}\right| \geqslant\left|f_{n}\right|, \quad n \geqslant m, m \in \mathfrak{R}, \\
\left|f_{m}\right| e^{\tau \mu_{m}^{1 / 2}} \geqslant\left|f_{n}\right| e^{\tau \mu_{n}^{1 / 2}}, \quad n \leqslant m, m \in \mathfrak{R} .
\end{gathered}
$$

Again we let

$$
\Phi_{m, k}(x ; f)=\sum_{n=1}^{\infty}\left[\frac{4 \mu_{m} \mu_{n}}{\left(\mu_{m}+\mu_{n}\right)^{2}}\right]^{k} f_{n} u_{n}(x),
$$

so that for $m \in \Re$

$$
\begin{aligned}
\frac{\Phi_{m, k}(x ; f)}{f_{m}^{*} U(x)} & =u_{m}^{*}(x)+\left(\sum_{n=1}^{m-1}+\sum_{n=m+1}^{\infty}\right)\left[\frac{4 \mu_{m} \mu_{n}}{\left(\mu_{m}+\mu_{n}\right)^{2}}\right]^{k} \frac{f_{n} u_{n}(x)}{\alpha_{m} f_{m} U(x)} \\
& =u_{m}^{*}(x)+T_{1}(x)+T_{2}(x),
\end{aligned}
$$

where $T_{1}$ and $T_{2}$ denote the finite and infinite sums, respectively. As in $\S 3$, we are going to show that $T_{1}(x), T_{2}(x) \rightarrow 0$ uniformly on $(a, b)$ as $m \rightarrow \infty, m \in \Re$, and where $k=\tilde{k}_{m}$ in the statement of Theorem 2. By $\left(\mathrm{A}_{7}\right)$ and (3.2) we will then have $V\left[\left(L^{\tilde{k_{m}}} f\right)(x)\right] \geqslant m$, which is the desired conclusion.

Beginning with $T_{2}(x)$, we first have

$$
\left|T_{2}(x)\right| \leqslant \sum_{n=m+1}^{\infty}\left[\frac{4 \mu_{m} \mu_{n}}{\left(\mu_{m}+\mu_{n}\right)^{2}}\right]^{k} \mu_{n}^{\beta+\delta},
$$

and this is identical to the sum in (3.4). The argument used to show $\left[S_{2}(x) / f_{m}^{*} U(x)\right]$ $\rightarrow 0$ hinged upon showing that

$$
x_{\max }=(k+h) /(k-h) \leqslant \mu_{m+1} / \mu_{m}
$$

and

$$
\mu_{m}^{1+h} g\left(\mu_{m+1} / \mu_{m}\right) \rightarrow 0
$$

The same argument will therefore work for $T_{2}(x)$ as long as we can establish (4.3) and (4.4) for the set of hypotheses (2.10)-(2.12) and where $k=\tilde{k}_{m}$. 
Statement (4.3) is equivalent to

$$
h\left(\mu_{m}+\mu_{m+1}\right) \leqslant k \theta_{m}
$$

as the paragraph below (3.5) points out. Now

$$
\theta_{m}=\mu_{m+1}-\mu_{m}=\left(\mu_{m+1}^{1 / 2}-\mu_{m}^{1 / 2}\right)\left(\mu_{m+1}^{1 / 2}+\mu_{m}^{1 / 2}\right)=\omega_{m}\left(\mu_{m+1}^{1 / 2}+\mu_{m}^{1 / 2}\right) \geqslant 2 K \mu_{m}^{1 / 2}
$$

by (2.10). Thus (4.5) is implied by $2 h \mu_{m+1} \leqslant 2 K d_{m} \mu_{m}^{3 / 2}$ (where we have put $\left.k=\tilde{k}_{m}\right) \Leftrightarrow d_{m} \mu_{m}^{1 / 2} \geqslant\left[h \mu_{m+1} / K \mu_{m}\right]$, and this is condition (2.11). Therefore (4.3) holds.

Working with (4.4),

$$
\begin{aligned}
\mu_{m}^{1+h} g\left(\mu_{m+1} / \mu_{m}\right) & =\mu_{m}^{1+h}\left[\frac{4\left(\mu_{m+1} / \mu_{m}\right)}{\left(1+\left(\mu_{m+1} / \mu_{m}\right)\right)^{2}}\right]^{k}\left(\mu_{m+1} / \mu_{m}\right)^{h} \\
& =\mu_{m} \mu_{m+1}^{h}\left[\frac{4 \mu_{m} \mu_{m+1}}{\left(\mu_{m}+\mu_{m+1}\right)^{2}}\right]^{k}<\mu_{m+1}^{1+h}\left[\frac{4 \mu_{m} \mu_{m+1}}{\left(\mu_{m}+\mu_{m+1}\right)^{2}}\right]^{k} \\
& =\mu_{m+1}^{1+h}\left[\frac{2 \mu_{m}^{1 / 2} \mu_{m+1}^{1 / 2}}{\mu_{m}+\mu_{m+1}}\right]^{2 k}=\mu_{m+1}^{1+h}\left[\frac{2 \mu_{m}^{1 / 2}\left(\mu_{m}^{1 / 2}+\omega_{m}\right)}{\mu_{m}+\left(\mu_{m}^{1 / 2}+\omega_{m}\right)^{2}}\right]^{2 k} \\
& =\mu_{m+1}^{1+h}\left[\frac{2 \mu_{m}+2 \mu_{m}^{1 / 2} \omega_{m}}{2 \mu_{m}+2 \mu_{m}^{1 / 2} \omega_{m}+\omega_{m}^{2}}\right]^{2 k}=\mu_{m+1}^{1+h}\left[1-\frac{1}{2 \mu_{m}+2 \mu_{m}^{1 / 2} \omega_{m}+\omega_{m}^{2}}\right]^{2 k} \\
& =\mu_{m+1}^{1+h}\left[1-\frac{1}{E_{m}}\right]^{2 k}\left(E_{m}=\left(2 \mu_{m}+2 \mu_{m}^{1 / 2} \omega_{m}+\omega_{m}^{2}\right) / \omega_{m}^{2}\right) \\
& =\mu_{m+1}^{1+h}\left[\left[1-\frac{1}{E_{m}}\right]^{E_{m}}\right]^{\left(2 k / E_{m}\right)}<\mu_{m+1}^{1+h} e^{-\left(2 k / E_{m}\right)} .
\end{aligned}
$$

But putting $k=\tilde{k}_{m}=d_{m} \mu_{m}$ makes

$$
\frac{2 k}{E_{m}}=\frac{2 d_{m} \mu_{m} \omega_{m}^{2}}{\mu_{m}+\left(\mu_{m}^{1 / 2}+\omega_{m}\right)^{2}}=\frac{2 d_{m} \omega_{m}^{2}}{1+\left(1+\left(\omega_{m} / \mu_{m}^{1 / 2}\right)\right)^{2}} \geqslant \frac{d_{m} \omega_{m}^{2}}{3}
$$

by (2.10). Then (2.6) does not exceed $\mu_{m+1}^{1+h} \exp \left(-d_{m} \omega_{m}^{2} / 3\right)$, which tends to 0 by (2.12). This completes the proof that $T_{2}(x) \rightarrow 0$.

To show that the finite sum $T_{1}(x) \rightarrow 0$, we modify an argument of Pólya and Wiener [10, p. 253]. Observe that for positive $x$ and $x_{0}$,

$$
\left(\frac{2 x_{0} x}{x_{0}^{2}+x^{2}}\right)^{2 k}=\left(\frac{x_{0}^{2}+x^{2}}{2 x_{0} x}\right)^{-2 k}=\left(\frac{x_{0}}{2 x}+\frac{x}{2 x_{0}}\right)^{-2 k} .
$$

Let $x_{0} / 2 x+x / 2 x_{0}=1+y$, so that $y=\left(x-x_{0}^{2}\right) /\left(2 x x_{0}\right)$. The condition $y<1$ is equivalent to $x^{2}-4 x x_{0}+x_{0}^{2}<0$. In particular, if we require $x_{0}(2-\sqrt{3})<x<x_{0}$, 
then we shall have $y<1$. The quantity (4.7) may then be replaced by

$$
(1+y)^{-2 k}<\left[e^{-y / 2}\right]^{2 k}=\left[e^{-y}\right]^{k} \quad(0<y<1) .
$$

If there are any integers $n \leqslant m-1$ for which $\mu_{n}^{1 / 2} \geqslant \mu_{m}^{1 / 2}(2-\sqrt{3})$, let us say that they take up the range $m_{1} \leqslant n \leqslant m-1$. Then

$$
\left|T_{1}(x)\right| \leqslant\left(\sum_{n=1}^{m_{1}-1}+\sum_{n=m_{1}}^{m-1}\right)\left[\frac{4 \mu_{m} \mu_{n}}{\left(\mu_{m}+\mu_{n}\right)^{2}}\right]^{k}\left[\frac{f_{n} u_{n}(x)}{f_{m}^{*} U(x)}\right]=R_{1}(x)+R_{2}(x),
$$

with $R_{1}(x)$ denoting the sum over $1 \leqslant n \leqslant m_{1}-1$ and $R_{2}(x)$ the sum over $m_{1} \leqslant n \leqslant m-1$. If the sum defining $R_{1}(x)$ is empty we take $m_{1}=1$. The first factor in $R_{2}(x)$ satisfies, in view of (4.8),

$$
\begin{aligned}
{\left[\frac{4 \mu_{m} \mu_{n}}{\left(\mu_{m}+\mu_{n}\right)^{2}}\right]^{k} } & =\left[\frac{2 \mu_{m}^{1 / 2} \mu_{n}^{1 / 2}}{\mu_{m}+\mu_{n}}\right]^{2 k}=\left[1+\frac{\left(\mu_{m}^{1 / 2}-\mu_{n}^{1 / 2}\right)^{2}}{2 \mu_{m}^{1 / 2} \mu_{n}^{1 / 2}}\right]^{-2 k} \\
& <\exp \left(-\frac{k\left(\mu_{m}^{1 / 2}-\mu_{n}^{1 / 2}\right)^{2}}{2 \mu_{m}^{1 / 2} \mu_{n}^{1 / 2}}\right) .
\end{aligned}
$$

Making use of (4.1), we have for $m \in \mathfrak{N}$,

$$
\begin{aligned}
\left|R_{2}(x)\right| \leqslant & \sum_{n=m_{1}}^{m-1} \mu_{n}^{\beta+\delta} e^{\tau\left(\mu_{m}^{1 / 2}-\mu_{n}^{1 / 2}\right)} \exp \left(-\frac{k\left(\mu_{m}^{1 / 2}-\mu_{n}^{1 / 2}\right)^{2}}{2 \mu_{m}^{1 / 2} \mu_{n}^{1 / 2}}\right) \\
& <\mu_{m}^{\beta+\delta} \sum_{n=m_{1}}^{m-1} \exp \left\{\left(\mu_{m}^{1 / 2}-\mu_{n}^{1 / 2}\right)\left[\tau-\frac{k\left(\mu_{m}^{1 / 2}-\mu_{n}^{1 / 2}\right)}{2 \mu_{m}^{1 / 2} \mu_{n}^{1 / 2}}\right]\right\} .
\end{aligned}
$$

Next,

$$
\frac{\mu_{m}^{1 / 2}-\mu_{n}^{1 / 2}}{2 \mu_{m}^{1 / 2} \mu_{n}^{1 / 2}} \geqslant \frac{\mu_{m}^{1 / 2}-\mu_{m-1}^{1 / 2}}{2 \mu_{m}}=\frac{\omega_{m-1}}{2 \mu_{m}}
$$

and so the factor

$$
\tau-\frac{k\left(\mu_{m}^{1 / 2}-\mu_{n}^{1 / 2}\right)}{2 \mu_{m}^{1 / 2} \mu_{n}^{1 / 2}} \leqslant \tau-\frac{k \omega_{m-1}}{2 \mu_{m}}=\tau-\frac{d_{m} \omega_{m-1}}{2}
$$

is eventually negative; here we have put $k=\tilde{k}_{m}=d_{m} \mu_{m}$. Since

$$
\begin{aligned}
\mu_{m}^{1 / 2}-\mu_{n}^{1 / 2} & =\left(\mu_{m}^{1 / 2}-\mu_{m-1}^{1 / 2}\right)+\left(\mu_{m-1}^{1 / 2}-\mu_{m-2}^{1 / 2}\right)+\cdots+\left(\mu_{n+1}^{1 / 2}-\mu_{n}^{1 / 2}\right) \\
& \geqslant \omega_{m-1}+\omega_{m-2}+\cdots+\omega_{n} \geqslant(m-n) K,
\end{aligned}
$$

by (2.10), then (4.9) is not greater than

$$
\begin{aligned}
& \mu_{m}^{\beta+\delta} \sum_{n=m_{1}}^{m-1} \exp \left(K(m-n)\left(\tau-\frac{d_{m} \omega_{m-1}}{2}\right)\right) \\
& \quad<\mu_{m}^{\beta+\delta} \sum_{j=1}^{\infty}\left\{\exp \left(K\left(\tau-\frac{d_{m} \omega_{m-1}}{2}\right)\right)\right\}^{j}=\mu_{m}^{\beta+\delta} F_{m} /\left(1-F_{m}\right)
\end{aligned}
$$


where $F_{m}=\exp \left(K\left(\tau-d_{m} \omega_{m-1} / 2\right)\right)$. Now

$$
F_{m}=e^{K \tau} \exp \left[-K d_{m} \omega_{m-1} / 2\right],
$$

and this tends to 0 by (2.12). This proves $\left|R_{2}(x)\right| \rightarrow 0$.

There remains the term $R_{1}(x)$, in which $\mu_{n}^{1 / 2} \leqslant \mu_{m}^{1 / 2}(2-\sqrt{3})$, and in which $y \geqslant 1$, where $y$ is defined below (4.7) with $x_{0}=\mu_{m}^{1 / 2}, x=\mu_{n}^{1 / 2}$. This being the case,

$$
\left[2 \mu_{m}^{1 / 2} \mu_{n}^{1 / 2} /\left(\mu_{m}+\mu_{n}\right)\right]^{2 k}=(1+y)^{-2 k}<(1 / 4)^{k}
$$

and therefore,

$$
\begin{aligned}
\left|R_{1}(x)\right| & \leqslant \sum_{n=1}^{m_{1}-1}(1 / 4)^{k} \mu_{m}^{\beta+\delta} \exp \left(\tau\left(\mu_{m}^{1 / 2}-\mu_{n}^{1 / 2}\right)\right) \\
& \leqslant m \mu_{m}^{\beta+\delta} \exp \left[-k \ln 4+\tau\left(\mu_{m}^{1 / 2}-\mu_{n}^{1 / 2}\right)\right] \\
& <m \mu_{m}^{\beta+\delta} \exp \left[-k \ln 4+\tau \mu_{m}^{1 / 2}\right] \\
& =m \mu_{m}^{\beta+\delta} \exp \left(\mu_{m}^{1 / 2}\left[\tau-(\ln 4) d_{m} \mu_{m}^{1 / 2}\right]\right) \quad\left(k=\tilde{k}_{m}\right) \\
& \leqslant m \mu_{m}^{\beta+\delta} \exp \left(-\mu_{m}^{1 / 2}\right) \quad(\text { by }(2.11))
\end{aligned}
$$

and this tends to 0 because $\mu_{m} \rightarrow \infty$. This completes the proof of Theorem 2 .

Corollary 2. Assume that the eigenvalues have the asymptotic form

$$
\mu_{n}=\alpha n^{2 q}(1+o(1)), \quad n \rightarrow \infty,
$$

where $q \geqslant 1$ and $\alpha$ is constant, and specifically $\mu_{n}=\alpha n^{2 q}\left(1+\varepsilon_{n}\right)^{2}$, where $((n+1) / n)^{4} \geqslant \varepsilon_{n} / \varepsilon_{n+1} \geqslant 1$. for all $n$, and $\varepsilon_{n} \rightarrow 0$. Let $f(x)$ be admissible, and suppose that for each $\varepsilon>0$ there is a constant $A>0$ such that $V\left[\left(L^{k} f\right)(x)\right] \leqslant$ $A k^{1 /(1+q+e)}$ for all $k$ sufficiently large. Then $\lim _{\sup _{n \rightarrow \infty}}\left|f_{n}\right| e^{\tau \mu_{n}^{1 / 2}}<\infty$ for all $\tau>0$.

Proof. The asymptotic constants $\varepsilon_{n}$ are behaved in such a way that $\omega_{n}>\sqrt{\alpha} q n^{q-1}$. If $d_{n}$ is selected so that $d_{n}>n^{1-q+\varepsilon_{1}}$, where $0<\varepsilon_{1}<\varepsilon$, then (2.10)-(2.12) are satisfied. Specifically, let $d_{n}$ satisfy

$$
n^{1-q+\varepsilon_{1}}<d_{n}<\frac{n^{1-q+\varepsilon}}{\alpha\left(1+\varepsilon_{n}\right)^{2} A^{1+q+\varepsilon}},
$$

which implies that $\tilde{k}_{n}=d_{n} \mu_{n}=d_{n} \alpha n^{2 q}\left(1+\varepsilon_{n}\right)^{2}$ satisfies

$$
\alpha\left(1+\varepsilon_{n}\right)^{2} n^{1+q+\varepsilon_{1}}<\tilde{k}_{n}<(n / A)^{1+q+\varepsilon} .
$$

Hence, there are choices of $d_{n}$ leading to integer values of $\tilde{k}_{n}$. This value of $\tilde{k}_{n}$ gives

$$
V\left[\left(L^{\tilde{k}_{n}} f\right)(x)\right] \leqslant A\left(\tilde{k}_{n}\right)^{1 /(1+q+\varepsilon)}<A(n / A)=n
$$

which is at variance with Theorem 2 unless $\lim \sup _{n \rightarrow \infty}\left|f_{n}\right| e^{\tau \mu_{n}^{1 / 2}}<\infty$. This completes the proof.

For $q=1$ we again achieve, to within $\varepsilon$, the rate $k^{1 / 2}$ for the frequency of oscillation of $V\left[\left(L^{k} f\right)(x)\right]$, this time for the Legendre and Jacobi polynomials as well as all regular Sturm-Liouville problems. 
For the case of double eigenvalues, we modify the proofs above by writing $\Phi_{m, k}(x ; f)$ in the form

$$
\Phi_{m, k}(x ; f)=\sum_{n=1}^{\infty}\left[\frac{4 \mu_{m} \mu_{n}}{\left(\mu_{m}+\mu_{n}\right)^{2}}\right]^{k}\left[f_{n} u_{n}(x)+\tilde{f}_{n} \tilde{u}_{n}(x)\right],
$$

where $u_{n}(x)$ and $\tilde{u}_{n}(x)$ are independent normalized eigenfunctions corresponding to $\mu_{n}$. The sum in (4.11) is then understood to be over distinct eigenvalues. Step (3.3) is replaced by

$$
\begin{aligned}
\frac{\Phi_{m, k}(x ; k)}{g_{m}^{*} U(x)}= & \frac{u_{m}^{*}(x)+\sigma_{m} u_{m}^{*}(x)}{U(x)} \\
& +\sum_{n \neq m}\left[\frac{4 \mu_{m} \mu_{n}}{\left(\mu_{m}+\mu_{n}\right)^{2}}\right]^{k} \frac{\left[f_{n} u_{n}(x)+\tilde{f}_{n} \tilde{u}_{n}(x)\right]}{g_{m}^{*} U(x)}
\end{aligned}
$$

where $g_{m}$ is either $f_{m}$ or $\tilde{f}_{m}$, one of which will belong to the subsequence of coefficients determined by $\Re ; \sigma_{m}$ stands for either $f_{m} / \tilde{f}_{m}$ or its inverse, and the roles of $u_{n}(x)$ and $\tilde{u}_{n}(x)$ in (4.12) may be reversed from (4.11). In either case, $\left(\mathrm{A}_{7}\right)$ assures that the first term on the right of (4.12) has strongly separated zeros. We treat the other terms as before, except that there are now two factors $\mu_{n}^{\beta+\delta}$ in (3.4), and similarly for $S_{1}(x)$.

5. Analytic expansions. We shall assume in this section that the complex differential equation $p_{2}(z) y^{\prime \prime}+p_{1}(z) y^{\prime}+p_{0}(z) y=0$ has a basis of solutions each of which is analytic in a region $\Omega$ containing the interval $\langle a, b\rangle$. We fix a base point $x_{0} \in(a, b)$ and consider the initial value problems

$$
\begin{aligned}
& \tilde{L} y=-P(z) \lambda y, \quad y\left(x_{0}, \lambda\right)=1, \quad y^{\prime}\left(x_{0}, \lambda\right)=0, \\
& \tilde{L} y=-P(z) \lambda y, \quad y\left(x_{0}, \lambda\right)=0, \quad y^{\prime}\left(x_{0}, \lambda\right)=1 .
\end{aligned}
$$

Denote the solutions of (5.1) and (5.2) by $\theta(z, \lambda)$ and $\phi(z, \lambda)$, respectively. These solutions are valid for $z \in \Omega$ and all complex $\lambda$.

The more standard form of (1.2) is

$$
-\left(p(x) y^{\prime}\right)^{\prime}+q(x) y=\lambda \rho(x) y .
$$

This may be brought into Liouville normal form by making the change of variables

$$
t=t(x)=\int_{x_{0}}^{x} \sqrt{\rho(s) / p(s)} d s, \quad w(t)=u(x)[\rho(x) p(x)]^{1 / 4}
$$

(see [2, p. 297]). Then (5.3) is equivalent to

$$
-w^{\prime \prime}(t)+Q(t) w(t)=\lambda w(t)
$$

where $Q$ depends on $q, p$ and $\rho$ evaluated at $x=x(t)$. Since $p_{2}(z)$ and $P(z)$ do not vanish in $\Omega$, then the solutions of (5.5) are analytic in the domain $\Omega_{t}$ which is the image of $\Omega$ under the map $t=t(x)$. Let $\Theta(t, \lambda)$ and $\Phi(t, \lambda)$, for complex $t$ and $\lambda$, correspond to the solutions $\theta(x, \lambda)$ and $\phi(x, \lambda)$ defined by (5.1) and (5.2). That is, $\Theta(t, \lambda)$ and $\Phi(t, \lambda)$ solve (5.5) and satisfy certain real initial conditions at $t\left(x_{0}\right)=0$. Classical arguments show that $\Theta(t, \lambda)$ and $\Phi(t, \lambda)$ are entire functions of $\lambda$ of order 
not more than $1 / 2[6,7,20]$. Moreover, if $\mathcal{K}_{t}$ is a compact subset of $\Omega_{t}$, then a constant $M$ can be found such that

$$
|\Theta(t, \lambda)| \leqslant e^{\left.M \lambda\right|^{1 / 2}}, \quad|\Phi(t, \lambda)| \leqslant e^{\left.M \lambda\right|^{1 / 2}}
$$

for all $t \in \mathcal{K}_{t}$ and all complex $\lambda$. Transforming conditions (5.6) back to the $z$-variable by means of (5.4), it follows that for each compact subset $\mathscr{K} \subset \Omega$, there exist constants $M_{1}$ and $M_{2}$ such that

$$
|\theta(z, \lambda)| \leqslant M_{1} e^{M_{2}|\lambda|^{1 / 2}}, \quad|\phi(z, \lambda)| \leqslant M_{1} e^{M_{2}|\lambda|^{1 / 2}}
$$

for all $z \in \mathscr{K}$ and all $\lambda$.

Now the eigenfunctions $u_{n}(x)$ may be conveniently expressed in terms of $\theta(x, \lambda)$ and $\phi(x, \lambda)$. We have, in fact,

$$
u_{n}(z)=u_{n}\left(x_{0}\right) \theta\left(z, \mu_{n}\right)+u_{n}^{\prime}\left(x_{0}\right) \phi\left(z, \mu_{n}\right)
$$

for all $z \in \Omega$ and $n=1,2,3, \ldots$ Using the estimates (5.7) in (5.8), we have, for an arbitrary compact set $\mathscr{K} \subset \Omega$ and $z \in \mathcal{K}$,

$$
\left|u_{n}(z)\right| \leqslant\left(\mu_{n}^{\beta}+\mu_{n}^{\gamma}\right) U\left(x_{0}\right) M_{1} \exp \left[M_{2}|\lambda|^{1 / 2}\right],
$$

where we have used $\left(\mathrm{A}_{5}\right)$.

THEOREM 3. Suppose that $f(x)$ is admissible and satisfies $\lim \sup _{n \rightarrow \infty}\left|f_{n}\right| e^{\tau \mu_{n}^{1 / 2}}<\infty$, for every $\tau>0$. Then the eigenfunction expansion (1.4) converges uniformly on compact subsets of $\Omega$. Thus $f(x)$ is analytically continuable into $\Omega$.

Proof. Given a compact set $\mathcal{K} \subset \Omega$, we know from (5.9) that constants $M_{1}$ and $M_{2}$ exist such that $\left|u_{n}(z)\right| \leqslant M_{1} \mu_{n}^{\beta+\delta} \exp \left[M_{2} \mu_{n}^{1 / 2}\right]$ for $z \in \mathscr{K}$ and all $n$. Choose $\tau>M_{2}$ and find a constant $M_{3}$ so that $\left|f_{n}\right| \leqslant M_{3} \exp \left[-\tau \mu_{n}^{1 / 2}\right]$ for all $n$. Then

$$
\left|f_{n} u_{n}(z)\right| \leqslant M_{1} M_{3} \mu_{n}^{\beta+\delta} \exp \left[\left(M_{2}-\tau\right) \mu_{n}^{1 / 2}\right] .
$$

Certainly $\exp \left[\left(M_{2}-\tau\right) \mu_{n}^{1 / 2}\right]<\mu_{n}^{-(\beta+\delta+2)}$ for $n$ large enough, and when this is inserted into (5.10) it follows that $\sum_{n=1}^{\infty}\left|f_{n} u_{n}(z)\right| \leqslant M_{1} M_{3} \Sigma_{1}^{\infty}(1 / n)^{2}$. Therefore $\sum_{n=1}^{\infty} f_{n} u_{n}(z)$ is analytic in $\Omega$, and this affords the analytic continuation of $f(x)$ into $\Omega$.

COROLlaRY 3. Suppse the eigenvalues $\mu_{n}$ satisfy the hypotheses of either Corollary 1 or 2 ; that is, suppose $\mu_{n}=\alpha n^{p}(1+o(1))(p \geqslant 1)$ or $\mu_{n}=\alpha n^{2 q}(1+o(1))(q \geqslant 1)$. Let $f(x)$ be an admissible function which satisfies the corresponding assumption on $V\left[\left(L^{k} f\right)(x)\right]$ in one of the corollaries; i.e., either $V\left[\left(L^{k} f\right)(x)\right] \leqslant A k^{1 /(1+p+\varepsilon)}$ or $V\left[\left(L^{k} f\right)(x)\right] \leqslant A k^{1 /(1+q+\varepsilon)}$. Then $f(x)$ is the restriction to $(a, b)$ of a function analytic in $\Omega$.

When $p=q=1$, we have in both cases $V\left[\left(L^{k} f\right)(x)\right] \leqslant A k^{1 /(2+\varepsilon)}$. If either $\mu_{n}=\alpha n(1+o(1))$ or $\mu_{n}=\alpha n^{2}(1+o(1))$, this condition implies that $f(x)$ is analytic in $\Omega$. Eigenvalues for the classical orthogonal polynomial problems, as well as all regular Sturm-Liouville problems, fall into one of these categories. Apart from the factor $\varepsilon$, then, Corollary 3 is a generalization of Hille's Theorem $B$.

For the classical orthogonal polynomials, $\Omega$ is the complex plane with $\{a\}$ and $\{b\}$ removed (since the solutions are polynomials, the nonsimple connectivity of $\Omega$ is of 
no consequence). Hence, the conclusion of Corollary 3 is that $f(x)$ is analytic everywhere except possibly for $x=a$ and $x=b$. But the eigenfunctions $u_{n}(z)$ are now entire functions, and so (5.10) must hold in a neighborhood of $z=b$ if it holds in, say, an annulus centered at $z=b$. This rules out any singularity at $z=b$, if it is finite, and similarly at $z=a$. Thus $f(x)$ is an entire function.

\section{REFERENCES}

1. M. Abramowitz and I. Stegun (eds.), Hardbook of mathematical functions with formulas, graphs and mathematical tables, Nat. Bur. Standards Appl. Math. Ser., no. 55, Superintendent of Documents, U. S. Government Printing Office, Washington, D.C., 1964. MR29 \#4914.

2. G. Birkhoff and G.-C. Rota, Ordinary differential equations, Wiley, New York, 1969.

3. R. P. Boas and C. Prather, Final sets for operators on finite Fourier transforms, Houston Math. J. 5 (1979), 29-36.

4. E. A. Coddington and N. Levinson, Theory of ordinary differential equations, McGraw-Hill, New York, 1955.

5. E. Hille, On the oscillation of differential transform. II, Trans. Amer. Math. Soc. 52 (1942), 463-497.

6. , Lectures on ordinary differential equations, Addison-Wesley, Reading, Mass., 1969.

7. _ Differential equations in the complex domain, Wiley, New York, 1976.

8. V. E. Kacnel'son, On the oscillation of derivatives of almost periodic functions, Teor. Funkcii Funkcional. Anal. i Priložen 2 (1966), 42-54. (Russian)

9. G. Pólya, On the zeros of the derivatives of a function and its analytic character, Bull. Amer. Math. Soc. 49 (1943), 178-191.

10. G. Pólya and N. Wiener, On the oscillations of the derivatives of a periodic function, Trans. Amer. Math. Soc. 52 (1942), 245-256.

11. C. Prather, On some old and new theorems on final sets, Houston Math. J. 7 (1981), 407-430.

12. Final sets for operators on classes of entire functions representable as a Fourier integral, J. Math. Anal. Appl. 82 (1981), 200-220.

13. _ The oscillation of derivatives. The Bernstein problem for Fourier integrals, submitted.

14. The oscillation of differential transforms. The Bernstein problem for Hermitian and Laguerre expansions, preprint.

15. J. K. Shaw, On the oscillatory behavior of singular Sturm-Liouville expansions, Trans. Amer. Math. Soc. 257 (1980), 483-505.

16. J. K. Shaw and C. L. Prather, Zeros of successive derivatives of functions analytic in a neighborhood of a single pole, Michigan Math. J. 29 (1982), 111-119.

17. A A Pólya "shire" theorem for functions with algebraic singularities, Internat. J. Math. Math. Sci. 5 (1982), 691-706.

18. G. Szegö, On the oscillation of differential transforms. I, Trans. Amer. Math. Soc. 52 (1942), 450-462.

19. On the oscillation of differential transform. IV, Trans. Amer. Math. Soc. 53 (1943), 463-468.

20. E. C. Titchmarsh, Eigenfunction expansions associated with second order differential equations. Part I, Clarendon Press, Oxford, 1962.

Department of Mathematics, Virginia Polytechnic Institute and State University, BlacksBURG, VIRGINIA 24061 